\title{
Typical Hesitant Fuzzy Sets - Evaluating Strategies in GDM Applying Consensus Measures
}

\author{
Monica Matzenauer ${ }^{a}$ and Renata Reiser ${ }^{a}$ and Helida Santos ${ }^{b}$ and Benjamin Bedregal ${ }^{c}$ \\ ${ }^{a}$ PPGC, CDTEC, UFPel, Pelotas - RS, Brazil, \{monica.matzenauer,reiser $\}$ @inf.ufpel.edu.br \\ ${ }^{b}$ PPGComp, C3, FURG, Rio Grande - RS, Brazil, helida@ furg.br \\ ${ }^{c}$ PPgSC, DIMAP, UFRN, Natal - RN, Brazil, bedregal@ dimap.ufrn.br
}

\begin{abstract}
This work studies typical hesitant fuzzy sets (THFS) which can be used in group decision making (GDM) due to the possibility of more flexible values for expressions given by a group of decision makers, instead of using only one value resulting from aggregation expressing their preferences. Besides, formal definitions on fuzzy sets and hesitant fuzzy sets are presented. Moreover, it is considered its relations in THFS and also properties related with consensus measures.
\end{abstract}

Keywords: Typical Hesitant Fuzzy Sets, Consensus Measure, Group Decision Making.

\section{Introduction}

The concept of fuzzy sets (FS) [38] has been broadly accepted and applied in many categories of decision making problems (DMP) because of the fact that everything is allowed to be a matter of degree in fuzzy logic (FL) [39]. The prominent characterization of a FS is considering membership degrees to the elements.

In real-life situations, decision making problems contain many kinds of uncertainties $[9,24]$. For the purpose of handling these uncertainties, the extensions of FS as well as their applications have been an increasing interest in recent years. Several extensions have been developed since FS were introduced [7], such as intutionistic fuzzy sets (IFS) working with the membership and non-membership functions [3], type-2 fuzzy sets (T2FS) permitting the membership to be a fuzzy set [11], type-n fuzzy sets [3], fuzzy multisets considering elements which can be repeated more than once and hesitant fuzzy sets (HFS), when the membership function is expressed as a set of possible values [31].

HFS can be applied in many decision making problems in order to get a good alternative in DMP with multiple attributes and multiple persons, there are usually two ways: (1) aggregate the decision makers opinions under each attribute for alternatives, then aggregate the collective values of attributes for each alternative; (2) aggregate the attribute values given by the decision makers for each alternative, and then aggregate the decision makers opinions for each alternative [34].

Aggregation functions such as the mean, median or OWA (ordered weighted average) operators are used in a wide range of decision making contexts to summarize a set of inputs in a single output [4]. They can be used to provide an overall rating for an item or candidate based on multiple criteria, or to combine the preferences of experts into a single group evaluation. In some situations, it may also be informative to have an idea of whether the inputs agree with one another, or whether the aggregated score is the result of a compromise between a number of disparate subgroups.

Multi-criteria decision making (MCDM) refers to evaluating, prioritizing or selecting over some available alternatives $\left\{A_{1}, A_{2}, \ldots, A_{p}\right\}$ with respect to a set of criteria $\left\{c_{1}, c_{2}, \ldots, c_{q}\right\}$ which are usually conflicted with each other. In order to do that, it is necessary to assign a value to each alternative with respect to each criterion.

For instance, suppose some specialists $(\mathrm{M}, \mathrm{J}, \mathrm{W}, \mathrm{C})$ provide ratings for three styles of craft beers (shown in Table 1). As well as comparing the average, we can also see that while everyone more or less agrees that the craft beer Sour style is not very good, and the Pale Ale style is not too bad, there is a lack of consensus regarding the Weiss style.

\begin{tabular}{|c|cccc|c|}
\hline Craft beer style & M & J & W & C & Average \\
\hline \hline Sour & 0.3 & 0.2 & 0.2 & 0.4 & 0.275 \\
Weiss & 0.2 & 0.3 & 0.9 & 0.8 & 0.55 \\
Pale Ale & 0.65 & 0.7 & 0.65 & 0.6 & 0.65 \\
\hline
\end{tabular}

Table 1: Individual ratings for craft beer styles.

Consensus measures, that is, functions which give an idea of how much the inputs agree with one another, have been employed increasingly in decision making contexts. Such measures have been used in voting and preference aggregation, for example to describe a set of voters and group them according to the similarity in their preferences [6].

Similar to the standard divergence of the mean in statistical summaries, consensus measures can provide an indication of reliability or the degree to which an entirely evaluation reflects the opinions of a group. As such, they have also been used to inform consensus reaching processes [6], where a minimum level of consensus can be set and a final decision may not be accepted if the consensus measure output is below this threshold. The consensus level between the pairwise preferences of an individual and the group can also be used to make recommendations that will increase the overall agreement between experts. 
In [21], we find a review on decision making methods based on fuzzy aggregation operators from 1986 to 2017, also including HFS. However, it is clear that few works in the literature formally define consensus on HFS. So, despite the many studies in HFS and consensus measures for HFS, the present paper is focused on THFS.

In this sense, this revision summarizes properties from selected papers in order to underground future work and contribute with a formal definition of consensus measures on THFS, making use of aggregation operators and fuzzy connectives acting on Typical Hesitant Fuzzy Elements.

This paper is organized as follows: In Section 2 the notion of fuzzy sets together with fuzzy negations and other properties are provided. Definitions of aggregation operators, fuzzy preference relations and fuzzy consensus measure are also given. The notion of hesitant fuzzy sets together with hesitant fuzzy negations and aggregation operators in HFS are reported in Section 3. Definitions of preferences relations and consensus measure in HFS is also presented, as well as some selected articles already published in the literature, in which consensus measure is applied in the HFS context. Section 4 studies the definition of THFS including the definition of admissible order on those sets. Final remarks are presented in Section 5 .

\section{Fuzzy Sets and Consensus Measures}

We start with some definitions adopted on fuzzy sets, fuzzy preferences relations and fuzzy consensus measure.

\subsection{Fuzzy Sets}

FS theory has been widely applied in a diversity of areas to handle uncertainty. Let $U=[0,1]$ be the set of all fuzzy values and $\leq_{U}$ be the usual partial order of real numbers on $U$. Some important concepts are:

Definition 1 [7, Def. 2.1] A (type-1) fuzzy set $A$ on $\mathcal{X} \neq \emptyset$ is a mapping $A: \mathcal{X} \rightarrow U$, where a real number $A(\mathrm{x}) \in U$ is the membership degree of an element $\mathrm{x} \in \mathcal{X}$ in a FS $A$.

\subsection{Fuzzy Negations and Dual Functions}

Definition 2 (Fuzzy negation [6, Def. 5]). A function $N: U \rightarrow$ $U$ is called a fuzzy negation if it verifies:

N1: $N(0)=1$ and $N(1)=0$ (boundary conditions); and

N2: If $x \geq y$ then $N(x) \leq N(y), \forall x, y \in U$ (monotone nonincreasing property).

In addition, fuzzy negations which also satisfy the involutive property (N3) are called strong fuzzy negations (SFN).

N3: $N(N(x))=x, \forall x \in U$.

A fuzzy negation is strict if it is continuous and strictly decreasing. All SFN are strict and the more representative SFN is the Zadeh's negation that is given by $N_{S}(x)=1-x$.
Let $N$ be a FN function and $f: U^{n} \rightarrow U$ be a real function. The $N$-dual function of $f$ is denoted by $f_{N}: U^{n} \rightarrow U$ and defined as:

$$
f_{N}\left(x_{1}, \ldots, x_{n}\right)=N\left(f\left(N\left(x_{1}\right), \ldots, N\left(x_{n}\right)\right)\right) .
$$

By Eq.(1), $f$ and $f_{N}$ are dual functions. If $N$ is a SFN function, $\left(f_{N}\right)_{N}=f$, i.e, the $N$-dual function of $f_{N}$ coincides with $f$. Thus, it is clear that $f$ is a self-dual function.

\subsection{Aggregation Operators}

Definition 3 (Aggregation function [6, Def. 1]). A function $f$ : $U^{n} \rightarrow U, n>1$ is called an aggregation function if it is monotone non-decreasing in each argument and satisfies the boundary conditions $f(0, \ldots, 0)=0$ and $f(1, \ldots, 1)=1$.

Two distinct classes of aggregation functions are called triangular norms (t-norms) and triangular conorms (t-conorms).

Definition 4 [19] A t-(co)norm is a binary function $T(S): U^{2} \rightarrow U$ satisfying the commutativity, associativity, monotonicity and it has $1(0)$ as neutral element.

Additionally, let $\mathcal{F}_{\mathcal{X}}$ be the set of all FS on the universe $\mathcal{X} \neq \emptyset$. Thus $A \leq_{\mathcal{F}_{\mathcal{X}}} B$ if and only if $A(x) \leq B(x)$, for all $x \in \mathcal{X}$ and $A, B \in \mathcal{F}_{\mathcal{X}}$, and the following holds:

Proposition 1 [7, Prop. 2.1] Let T, $S: U^{2} \rightarrow U$ be a t-norm and t-conorm, respectively. $\left(\mathcal{F}_{\mathcal{X}}, \cup_{\mathcal{F}_{\mathcal{X}}}, \cap_{\mathcal{F}_{\mathcal{X}}}\right)$ is a complete lattice, where for all $A, B \in \mathcal{F}_{\mathcal{X}}$, union and intersection are, respectively, defined by: $\left(A \cup_{\mathcal{F}_{\mathcal{X}}} B\right)(x)=S\left(\mu_{A}(x), \mu_{B}(x)\right)$ and $\left(A \cap_{\mathcal{F}_{\mathcal{X}}} B\right)(x)=T\left(\mu_{A}(x), \mu_{B}(x)\right)$.

Example $1 S_{M}(x, y)=\max (x, y)$ is a t-conorm and $T_{M}(x, y)=\min (x, y)$ is a t-norm. $\left(S_{M}, T_{M}\right)$ is a pair of $N_{S}$-mutual dual aggregation functions. Moreover, union and intersection in this case can be, respectively, defined by: $\left(A \cup_{\mathcal{F}_{\mathcal{X}}} B\right)(x)=\max \left(\mu_{A}(x), \mu_{B}(x)\right)$ and $\left(A \cap_{\mathcal{F}_{\mathcal{X}}} B\right)(x)=$ $\min \left(\mu_{A}(x), \mu_{B}(x)\right)$.

So, $\left(U, \cup_{\mathcal{F}}, \cap_{\mathcal{F}}, 0,1\right)$ is a complete lattice of fuzzy values.

\subsection{Fuzzy Preference Relations}

For a finite set of alternatives, $\chi=\left\{x_{1}, x_{2} \ldots, x_{n}\right\}$, a fuzzy preference relation (FPR) is a fuzzy set on the product set $\chi \times \chi$ as follows.

Definition 5 [44, Def. 1] A FPR $\mathrm{R}$ on $\chi$ is characterized by a membership function $\mu_{\mathrm{R}}: \chi \times \chi \rightarrow U$.

Thus, the FPR $\mathrm{R}$ can be represented by an $n \times n$ matrix $[R]=$ $\left(r_{i j}\right)_{n \times n}$ where $r_{i j}=\mu_{\mathrm{R}}\left(x_{i}, x_{j}\right)$ for all $i, j \in\{1,2, \ldots, n\}$. In addition, an element $r_{i j}$ is interpreted as the preference degree of $x_{i}$ over $x_{j}$. If:

(i) $r_{i j}=0.5$, then it indicates indifference between $x_{i}$ and $x_{j}$ or maximal fuzziness;

(ii) $r_{i j}>0.5$, then $x_{i}$ is preferred to $x_{j}$;

(iii) $r_{i j}=1$, it implies that alternative $x_{i}$ is definitely preferred to alternative $x_{j}$ (crisp case); 
In some situations, it is required that $\mathrm{R}$ satisfies the additive reciprocity property:

$$
r_{i j}+r_{j i}=1 \quad i, j=1,2, \ldots n
$$

For instance, using the example in Table 1, it is possible to present the preference matrices for each specialist, related to all craft beer styles. Consider the preferences for the sour, weiss and pale ale styles are in this order in the rows and columns of each matrix. It can be noticed that for specialist M matrix, the sum of her preferences between the weiss and the sour styles has to be the value 1. For example, as M's preference value for the weiss style, over the sour, is 0.7 then the preference value for the sour style, over the weiss, is 0.3 , that it, the sum of the preferences between these two styles is equal to 1 . Analogously, this was also analyzed for all the other relations between styles.

$$
\begin{aligned}
& {\left[R^{1}\right]=\left(\begin{array}{lll}
0.500 & 0.600 & 0.316 \\
0.400 & 0.500 & 0.235 \\
0.684 & 0.765 & 0.500
\end{array}\right)} \\
& {\left[R^{2}\right]=\left(\begin{array}{lll}
0.500 & 0.400 & 0.222 \\
0.600 & 0.500 & 0.300 \\
0.778 & 0.700 & 0.500
\end{array}\right)} \\
& {\left[R^{3}\right]=\left(\begin{array}{lll}
0.500 & 0.182 & 0.235 \\
0.818 & 0.500 & 0.581 \\
0.765 & 0.419 & 0.500
\end{array}\right)} \\
& {\left[R^{4}\right]=\left(\begin{array}{lll}
0.500 & 0.333 & 0.400 \\
0.667 & 0.500 & 0.571 \\
0.600 & 0.429 & 0.500
\end{array}\right)}
\end{aligned}
$$

\subsection{Fuzzy Consensus Measure}

Consensus measures, that is, functions which give an overall idea of how much the inputs agree with one another, have been employed increasingly in GDM contexts.

As proposed in [6], consensus measures are defined by functions on the unit interval $[0,1]$ modelling the agreement related to several inputs based on two main properties. The first is the unanimity: interpreting the complete consensus which is achieved when all inputs are the same. The second is the minimal consensus, which is related to the special case of two inputs, resulting in a null-consensus whenever one of theses inputs lies at one of extremes (either 0 or 1 ) in the unit interval. The main idea of a consensus measure is formalized as follows:

Definition 6 [6, Def. 7] A function $C: U^{n} \rightarrow U$ is said to be a consensus measure if it satisfies:

C1: $C(a, a, \ldots, a)=1, \forall a \in U$ (Unanimity);

C2: $C(0,1)=C(1,0)=0($ Minimum consensus for $n=2)$.

Further desired properties of consensus measures [6]:

C3: $C\left(x_{1}, x_{2}, \ldots, x_{n}\right)=C\left(x_{\sigma(1)}, x_{\sigma(2)}, \ldots, x_{\sigma(n)}\right)$, for all $\sigma$ permutation on $\{1, \ldots, \mathrm{n}\}$ and $x \in U^{n}$ (Symmetry);

C4: $C\left(x_{1}, x_{2}, \ldots, x_{n}\right)=0$, when $n=2 k, k=\#\left\{x_{i}: x_{i}=0\right\}=$ $\#\left\{x_{i}: x_{i}=1\right\}$ (Maximum dissension);

C5: $C\left(x_{1}, x_{2}, \ldots, x_{n}\right)=C\left(N\left(x_{1}\right), N\left(x_{2}\right), \ldots, N\left(x_{n}\right)\right)$, when $N$ is a strong fuzzy negation (Reciprocity);
C6: $C(\mathbf{x})=C(\mathbf{x}, \mathbf{x})=C(\mathbf{x}, \mathbf{x}, \mathbf{x})$ and so on, $\forall \mathbf{x} \in U^{n}($ Replication invariance);

C7: For $n=2 k$, let half of the evaluations be equal and denoted as $\mathbf{a}=(a, a, \ldots, a) \in U^{k}$. If $\left|a-x_{j}\right| \leq\left|a-y_{j}\right|$ for $j=$ $1, \ldots, k$ then $C\left(a, x_{1}, x_{2}, \ldots, x_{k}\right) \geq C\left(a, y_{1}, y_{2}, \ldots, y_{k}\right)$. (Monotonicity w.r.t. majority).

There are many works related to consensus measure which have been employed increasingly in GDM contexts, in most cases fuzzy connectives and aggregation operators are considered, reporting the sets of membership degree which are obtained from specialist opinions. Such measures have been applied in voting preferences aggregation [1] and used to inform consensus reaching processes [16].

Consensus provides understanding by distinct ways in GDM contexts, briefly described considering [16] and [28]:

- The state of agreement in a group, meaning a common feeling between the individuals about the values in question. From this perspective, consensus has been denoted as a full and unanimous agreement, though it has been considered questionable if such state is possible in a real world context.

- Methodology to reach consensus, which is also related to the sense given above, but having an evolution, in which the group attest for consensus with respect to their testimonies. This evolution can be freely reached or facilitated by a special individual.

- Method in which decisions should be meant in multiperson settings, aiming to achieve the consent, not necessarily the agreement, of the individuals by arranging views of all parties involved to obtain a decision that will produce what will be useful to the entire group. It is not necessarily related to a particular individual who may give consent to, and not necessarily his first choice, but because, for example, he wants to cooperate with the group.

The first approaches of consensus reaching process started between the 40s and 50s, with two main contributions considered the start of participatory management in GDM [12].

Later, consensus theory is developed in a more general form in 1981. These initial formulations describe the formation of group consensus, but do not provide an adequate account of settled patterns of disagreement. Later, many models of consensus reaching have been proposed, notably in the domain of so called rational consensus. Then, in 1985, classical consensus approaches were given, where the notion of consensus has conventionally been understood in terms of strict and unanimous agreement. Other contributions in fuzzy consensus and GDM appear in [35].

It is possible to find different consensus approaches in the literature, according to different criteria as the reference domain, considering distinct consensus approaches.

A. Consensus measures focused on an expert set, where consensus degrees are obtained in three steps:

(i) for each pair of individuals, a degree of agreement as to their opinions between all the pairs of options are computed, 
(ii) these agreement degrees are combined to obtain a degree of agreement of each pair of individuals as to their preferences between pairs of options; and finally,

(iii) these agreement degrees are combined to obtain an agreement degree of pairs of individuals as to their preferences between pairs of options, which is the consensus degree of the group of experts.

B. Consensus measures can also focus on the alternative set considering three levels of FPR:

(i) level of preference, indicating the consensus degree existing among all the preference values attributed by the experts to a specific preference;

(ii) level of alternative, which allows us to measure the consensus existing over all the alternative pairs where a given alternative is present; and

(iii) level of preference relation, evaluating the social consensus, that is, the current consensus existing among all the experts about all the preferences.

According to [17], in order to guide the experts to change their preferences during the discussion process, the analysis of levels in preferences relation seems to be adequate in consensus process designs. In addition, four current trends in the field of consensus models were also discussed:

I. Adaptative consensus models, providing strategies adapting the number of changes in the GDM problem which are required to the experts in each round of consensus. Research focuses in managing distinct representations of preference relations and methodologies and guarantying convergence of adaptive consensus [22].

II Trust based consensus models, providing techniques to explore unsuitable specialists for the decision process in order to consider a subgroup of relevant specialist improving the achievement of solutions of problems in GDM [2];

III Dynamic and changeable consensus models, investigating situations where alternatives might change or disappear while experts are discussing/making decision [32];

IV Consensus models based on agent theory, as a tool to obtain alternatives based on anthologies providing an advanced representation of information for possible evaluation of alternatives analyzed by groups of specialists [23].

The use of these models is still in an early stage of development and several future challenges have still to be solved.

In [35], it is introduced an algorithm that can automatically modify the diverging individual FPR so as to reach an acceptable consensus, avoiding to make the specialists change their preferences and then, turning the decision more scientific and more efficient.

Example 2 A GDM can be described as follows: For $l=$ $1,2, \ldots, m$, suppose that $m$ decision makers $\mathbf{e}_{l}$ provide their individual FPR $R^{l}=\left(r_{i j}^{l}\right)_{n \times n}$ over the alternatives $x_{1}, x_{2}, \ldots x_{n}$, and let $\lambda=\left(\lambda_{1}, \lambda_{2}, . ., \lambda_{m}\right)^{T}$ be the weight vector of the decision makers $\mathbf{e}_{l}(l=1,2, . ., m)$ with the condition $\sum_{l=1}^{m} \lambda_{l}=1$ and $0 \leq \lambda_{l} \leq 1$. The next example applies the algorithm proposed in [35, Alg. 4.1], using data presented in Table 1.

As presented in Subsection 2.4, we consider four specialists $\mathbf{e}_{l}(l=1,2,3,4)$ providing ratings for three styles of craft beers
( $n=1,2,3)$, and their corresponding matrices of FPR about three craft beer styles. So, in this example we applied the algorithm [35, Alg. 4.1] to reach an acceptable group consensus, that is summarized in the following steps:

Step 1. Constructing multiplicative consistent FPR $\bar{R}^{l}=$ $\left(\bar{r}_{i j}^{l}\right)_{n \times n}$, from $R^{l}=\left(r_{i j}\right)_{3 \times 3}$, for $l=1, \ldots, 4$. Thus, for $i, j=1, \ldots, n$, we obtain the following expression:

$$
\bar{r}_{i j}^{l}=\frac{\sqrt[n]{\prod_{t=1}^{n}\left(r_{i t} r_{t j}\right)}}{\sqrt[n]{\prod_{t=1}^{n}\left(r_{i t} r_{t j}\right)}+\sqrt[n]{\prod_{t=1}^{n}\left(\left(1-r_{i t}\right)\left(1-r_{t j}\right)\right)}},
$$

The matrices $\bar{R}^{l}$ of preference relation are as follows:

$$
\begin{aligned}
& {\left[\overline{R^{1}}\right]=\left(\begin{array}{lll}
0.500 & 0.400 & 0.684 \\
0.600 & 0.500 & 0.765 \\
0.316 & 0.235 & 0.500
\end{array}\right)} \\
& {\left[\overline{R^{2}}\right]=\left(\begin{array}{lll}
0.500 & 0.600 & 0.778 \\
0.400 & 0.500 & 0.700 \\
0.222 & 0.300 & 0.500
\end{array}\right)} \\
& {\left[\overline{R^{3}}\right]=\left(\begin{array}{lll}
0.500 & 0.818 & 0.765 \\
0.182 & 0.500 & 0.420 \\
0.235 & 0.580 & 0.500
\end{array}\right)} \\
& {\left[\overline{R^{4}}\right]=\left(\begin{array}{lll}
0.500 & 0.667 & 0.600 \\
0.333 & 0.500 & 0.429 \\
0.400 & 0.571 & 0.500
\end{array}\right)}
\end{aligned}
$$

Step 2. Then, the individual FPR $\bar{R}^{l}(l=1,2,3,4)$ are aggregated into a group FPR $\bar{R}$ by Eq. (2). For convenience, let $s=0$,

$$
\begin{gathered}
\bar{R}^{l(0)}=\left(\bar{r}_{i j}^{l(0)}\right)_{n \times n}=\bar{R}^{l}=\left(\bar{r}_{i j}^{l}\right)_{n \times n}, \\
\bar{R}^{(0)}=\left(\bar{r}_{i j}^{(0)}\right)_{n \times n}=\bar{R}=\left(\bar{r}_{i j}\right)_{n \times n}, l=1,2,3,4 ; \text { and } \\
\bar{r}_{i j}=\frac{\prod_{l=1}^{m}\left(r_{i j}^{l}\right)^{\lambda_{l}}}{\prod_{l=1}^{m}\left(r_{i j}^{l}\right)^{\lambda_{l}}+\prod_{l=1}^{m}\left(1-r_{i j}^{l}\right)^{\lambda_{l}}}, i, j=1,2, \ldots n
\end{gathered}
$$

Then, we obtain the matrix $\bar{R}^{(0)}$ as follows:

$$
\bar{R}^{(0)}=\left(\begin{array}{lll}
0.500 & 0.634 & 0.712 \\
0.366 & 0.500 & 0.588 \\
0.288 & 0.412 & 0.500
\end{array}\right)
$$

Step 3. The deviation degree between each individual FPR $\bar{R}^{l(0)}$ and the group FPR $\bar{R}^{(0)}$ is done by Eq. (3):

$$
d\left(\bar{R}^{l(s)}, \bar{R}^{(s)}\right)=\frac{1}{n^{2}} \sum_{i=1}^{n} \sum_{j=1}^{n}\left|r_{i j}^{l(s)}-r_{i j}^{(s)}\right| .
$$

The results parameters are reported in the sequence:

$$
\begin{aligned}
& d\left(\bar{R}^{(0)},{\overline{R^{1}}}^{(0)}\right)=0.0975, \quad d\left(\bar{R}^{(0)},{\overline{R^{2}}}^{(0)}\right)=0.0473, \\
& d\left(\bar{R}^{(0)},{\overline{R^{3}}}^{(0)}\right)=0.0901, \quad d\left(\bar{R}^{(0)},{\overline{R^{4}}}^{(0)}\right)=0.0673 .
\end{aligned}
$$

As the algorithm indicates, without loss of generality, it is considered $\rho=0.05$. Thus: $d\left(\bar{R}^{(0)},{\overline{R^{1}}}^{(0)}\right)=0.0975>0.05$; $d\left(\bar{R}^{(0)},{\overline{R^{3}}}^{(0)}\right)=0.0901>0.05 ; d\left(\bar{R}^{(0)},{\overline{R^{4}}}^{(0)}\right)=$ $0.0673>0.05$. Then, step 4 should be performed. 
Step 4. Supposing a normalization $\eta=0.5$, then it is calculated again the terms $\bar{R}_{l}^{(1)}=\left(\bar{r}_{i j l}^{(1)}\right)_{n \times n}, l=1,3,4$ and $\bar{R}^{(1)}=\left(\bar{r}_{i j}^{(1)}\right)_{n \times n}$, where $\bar{R}_{l}^{(1)}=\bar{R}_{l}^{(0)}, l=1,3$ and $i, j, l=2$ :

$$
\begin{aligned}
\bar{r}_{i j l}^{(1)} & =\frac{\left(\bar{r}_{i j l}^{(0)}\right)^{(1-\eta)}\left(\bar{r}_{i j}^{(0)}\right)^{\eta}}{\left(\bar{r}_{i j l}^{(0)}\right)^{(1-\eta)}\left(\bar{r}_{i j}^{(0)}\right)^{\eta}+\left(1-\bar{r}_{i j l}^{(0)}\right)^{(1-\eta)}\left(1-\bar{r}_{i j}^{(0)}\right)^{\eta}} \\
r_{i j}^{(1)} & =\frac{\prod_{l=1}^{m}\left(r_{i j l}^{(1)}\right)^{\lambda_{l}}}{\prod_{l=1}^{m}\left(r_{i j l}^{(1)}\right)^{\lambda_{l}}+\prod_{l=1}^{m}\left(1-r_{i j l}^{(1)}\right)^{\lambda_{l}}}, i, j=1,2,3,4 .
\end{aligned}
$$

Thus, the following holds:

$$
\begin{aligned}
& {\left[{\overline{R^{1}}}^{(1)}\right]=\left(\begin{array}{lll}
0.500 & 0.518 & 0.698 \\
0.482 & 0.500 & 0.683 \\
0.302 & 0.317 & 0.500
\end{array}\right) ;} \\
& {\left[{\overline{R^{3}}}^{(1)}\right]=\left(\begin{array}{lll}
0.500 & 0.736 & 0.739 \\
0.264 & 0.500 & 0.504 \\
0.261 & 0.496 & 0.500
\end{array}\right) ;} \\
& {\left[{\overline{R^{4}}}^{(1)}\right]=\left(\begin{array}{lll}
0.500 & 0.651 & 0.658 \\
0.349 & 0.500 & 0.508 \\
0.342 & 0.492 & 0.500
\end{array}\right) ;} \\
& {\left[\bar{R}^{(1)}\right]=\left(\begin{array}{lll}
0.500 & 0.630 & 0.720 \\
0.370 & 0.500 & 0.600 \\
0.280 & 0.400 & 0.500
\end{array}\right) .}
\end{aligned}
$$

Then, let $s=1$. Returning to Step 3 , it is necessary to recalculate the deviation degree between each individual preference relation $\bar{R}_{l}^{(1)}$ and the group preference relation $\bar{R}^{(1)}$ by Eq. (3):

$$
\begin{aligned}
& d\left(\bar{R}^{(1)},{\overline{R^{1}}}^{(1)}\right)=0.0499, d\left(\bar{R}^{(1)},{\overline{R^{2}}}^{(1)}\right)=0.0473, \\
& d\left(\bar{R}^{(1)},{\overline{R^{3}}}^{(1)}\right)=0.0475, \quad d\left(\bar{R}^{(1)},{\overline{R^{4}}}^{(1)}\right)=0.0332 .
\end{aligned}
$$

Now, all of the deviation relations are less then 0.05, thus the acceptable consensus of the group is achieved.

\section{Approaches for Consensus Measures on $\mathbb{H}$}

In 2010, Torra [31] introduces the notion of the so-called Hesitant Fuzzy Sets (HFS) which are defined in terms of functions returning a set of membership degrees for each element in the domain $\chi \neq \emptyset$. HFS are represented as "a function $h$ that when applied to $\chi$ returns a subset of $[0,1]$." As well, Torra presents a Lemma that HFS, where $h(x)$ is a non-empty closed interval, are Intuitionistic Fuzzy Sets (IFS), and as a consequence, they are interval-valued fuzzy sets and also, fuzzy sets.

\subsection{Hesitant Fuzzy Sets}

Definition 7 [34, Def. 2] Let $\chi$ be a non-empty fixed set, a HFS on $\chi$ is determined by a function $h: \chi \rightarrow \wp(U)$.

Based on Def. 7, a HFS on $\chi$ is determined by a function $h$ that when applied to $\chi$ returns a subset of $U$.

Moreover, a HFS can also be constructed from a set of fuzzy set.

Definition 8 [31, Def. 5] Let $M=\left\{\mu_{1}, \ldots, \mu_{n}\right\}$ be a set of $n$ membership functions. The HFS associated with $M$ is given as $h_{M}: X \rightarrow \wp(U), h_{M}(x)=\{\mu(x): \mu \in M\}$
In the process of MCDM, HFS can be useful to handle situations where there is indecision among many possible values for the preferences over objects. In such case, $M$ represents the assessments of the experts for each alternative and $h_{M}$ the assessments of the set of experts. However, note that it only allows to recover those HFS whose memberships are given by sets of cardinality less than or equal to $n$.

Afterward, results from Xia and Xu's research [34] completed the original Torra's definition of HFS by including the mathematical representation of a HFS as follows

$$
E=\left\{\left\langle x, h_{E}(x)\right\rangle: x \in \chi\right\},
$$

where $h_{E}(x)$ is a set of some values in the unitary interval $[0,1]$, denoting the possible membership degree of an element $x \in \chi$ to the set $E$. For convenience, $h=h_{E}(x)$ is called the hesitant fuzzy element (HFE) of $E$ and $H=\left\{h_{E}(x): x \in \chi\right\}$, the set of all HFE of $E$. Despite the differences in their definitions, the concepts of HFS and HFE can be used indistinctly [24].

A HFS in $\mathbb{H}$ on $\chi$ is a set of subsets in the unitary interval [0,1], one set for each element of the reference set $X$. A HFE is one of such sets, the one for a particular $x \in \chi$.

Xu-Xia-partial order [37] compares two HFE of different cardinalities, assuming the pessimistic scenario where the decision makers expect unfavorable outcomes.

They repeat the shortest element of the HFE with lower cardinality until both HFE have the same cardinality (a kind of $\beta$ normalization according to [4]) and then, they order these elements and compare the greatest elements of both HFE and so on, which means comparing the elements until reaching the lowest of both HFE. Formally, this will be defined as follows and to do so, we denote $\mathbb{N}_{k}=\{1, \ldots, k\}$.

Based on [30, Def. 7], let $\mathbb{H}=\{X \subseteq U: X$ is finite, $X \neq \emptyset\}$. Given $X \in \mathbb{H}$, let $\sigma_{X}: \mathbb{N}_{\# X} \rightarrow X$ be mapping such that for any $i \in \mathbb{N}_{\#(X-1)}$, the following holds:

$$
\sigma_{X}(i)<\sigma_{X}(i+1) .
$$

The $\sigma$-permutation in Eq. (5) can also be obtained by taking an increase reordering (via permutation) of elements in $X$.

Consider the $n$-dimensional upper simplex, given as $L_{n}(U)=$ $\left\{\mathbf{x}=\left[x_{1}, \ldots, x_{n}\right] \in U^{n}: x_{1} \leq \ldots \leq x_{n}\right\}$. Based on [30, Def. 8], for any $n \in \mathbb{N}^{+}=\mathbb{N}-\{0\}$, when $m=\# X$, we define the function $\beta_{n}: \mathbb{H} \rightarrow L_{n}$ as follows:

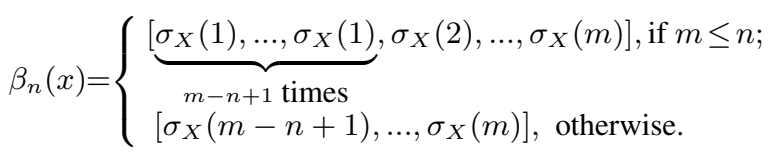

The partial order of $\mathrm{Xu}$-Xia denoted by $\leq_{X X}$ was reported in [29], as follows:

Definition 9 [30, Def. 9] Given $X, Y \in \mathbb{H}$, we say

$$
X \leq_{X X} Y \Leftrightarrow \beta_{n}(X) \leq_{L_{n}} \beta_{n}(Y) \text {, with } n=\max (\# X, \# Y) .
$$

According with [30, Prop.1], $\left(\mathbb{H}, \leq_{X X}\right)$ is a complete lattice with the bottom and top elements given by $0_{\mathbb{H}}=\{0\}$ and $1_{\mathbb{H}}=\{1\}$, respectively. 
HFS have been widely studied and applied because it is able to manage multilevels of uncertatity, mainly when dealing with situations in which the decision makers are hesitant about alternatives and linguistic variables needed to express their preferences. Main related works, consolidate applications in science and possible new perspectives are revised in systematic reviews, presenting recent and relevant literature of HFS, such as [7], [18] and [21].

In the next section it is also shown selected papers concerning HFS, mainly the ones which are related to THFS and/or to Consensus Measures approaches.

\subsection{Hesitant Fuzzy Negations}

Definition 10 [29] Let $\mathcal{N}: \mathbb{H} \rightarrow \mathbb{H}$ be a function. $\mathcal{N}$ is a hesitant fuzzy negation (HFN) if

$(\mathcal{N} 1) \mathcal{N}\left(0_{\mathbb{H}}\right)=1_{\mathbb{H}}$ and $\mathcal{N}\left(1_{\mathbb{H}}\right)=0_{\mathbb{H}}$ (boundary condition);

$(\mathcal{N} 2) \quad X \leq_{X X} Y \Rightarrow \mathcal{N}(Y) \leq_{X X} \mathcal{N}(X)$ (decreasing).

A HFN $\mathcal{N}$ is strong if it is involutive, i.e. if for each $X \in \mathbb{H}$ it satisfies: $(\mathcal{N} 3) \mathcal{N}(\mathcal{N}(X))=X$.

\subsection{Aggregation Operators on HFSs}

Definition 11 Let $\leq_{\mathbb{H}}$ be a partial order on $\mathbb{H}$. A function $\mathcal{A}: \mathbb{H}^{n} \rightarrow \mathbb{H}$ is an n-ary hesitant aggregation function w.r.t. $\leq_{\mathbb{H}},\left\langle\mathbb{H}, \leq_{\mathbb{H}}\right\rangle$-aggregation function in short, if the isotonicity and boundary conditions hold, $\forall i=1, \ldots, n$ :

(A1) $\mathcal{A}\left(X_{1}, \ldots, X_{n}\right) \leq_{\mathbb{H}} \mathcal{A}\left(Y_{1}, \ldots, Y_{n}\right)$, when $X_{i} \leq_{\mathbb{H}} Y_{i} ;$

$(\mathcal{A} 2) \mathcal{A}\left(\mathbf{0}_{\mathbb{H}}, \ldots, \mathbf{0}_{\mathbb{H}}\right)=\mathbf{0}_{\mathbb{H}}$ and $\mathcal{A}\left(\mathbf{1}_{\mathbb{H}}, \ldots, \mathbf{1}_{\mathbb{H}}\right)=\mathbf{1}_{\mathbb{H}}$.

Analogously, the extension of the notion of t-norms and tconorms for THFS can also be done. Thus,

Definition 12 [30, Def. 12] A function $\mathcal{T}, \mathcal{S}: \mathbb{H} \times \mathbb{H} \rightarrow \mathbb{H}$ is a typical hesitant triangular (co)norm, $\mathbb{H}-t$-(co)norm in short, if commutativity, associativity, monotonicity hold and it has $\mathbf{1}_{\mathbb{H}}\left(\mathbf{0}_{\mathbb{H}}\right)$ as neutral element.

Torra, in [31], proposed a definition of union and intersection for Hesitant Fuzzy Sets (HFS) that extends those by Zadeh. Given $A, B \in H F S(\chi)$ and where $\mathrm{S}$ and $\mathrm{T}$ are a t-conorm and a t-norm respectively, it is defined that:

$\left(A \cup_{\mathbb{H}} B\right)(x)=\{t \in A(x) \cup B(x): t \geq S(\inf A(x), \inf B(x))\} ;$ $\left(A \cap_{\mathbb{H}} B\right)(x)=\{t \in A(x) \cup B(x): t \leq T(\sup A(x), \sup B(x))\}$.

By [7], $\left(\mathbb{H}(X), \cup_{\mathbb{H}}, \cap_{\mathbb{H}}\right)$ is not a lattice. If for e.g. $X=$ $\{x\}$. Consider the HFS $A=\{0.3,0.5,0.6\}$ and $B=$ $\{0.4,0.5,0.7,0.8\}$, then $A \cap_{H F} B=\{0.3,0.4,0.5,0.6\}$ and so $A \cup_{\mathbb{H}}(A \cap \mathbb{H} B)=\{0.3,0.4,0.5,0.6\}$ which is different from $A$. So, $A \cup_{\mathbb{H}}(A \cap \mathbb{H} B) \neq A$, and the law of absorption does not hold.

\subsection{Preference Relations on Hesitant Fuzzy Sets}

The concept of HFS has shown itself to be useful in GDM problems. The definition of Hesitant Fuzzy Preference Relation (HFPR) was extended in [36].
Definition 13 [27, Def. 5] Let $X=\left\{x_{1}, x_{2}, \ldots, x_{n}\right\}$ be a fixed set. A HFPR $H$ on $X$, represented by $H=\left(h_{i j}\right)_{n \times n} \subset X \times X$ where $h_{i j}=\left\{h_{i j}^{\beta}, \beta=1,2, \ldots, \# h_{i j}\right\}$ (\#h $h_{i j}$ is the number of values in $h_{i j}$ ) is a HFE indicating all the possible preference degree(s) of alternative $x_{i}$ over $x_{j}$. Additionally, for $i, j=$ $1, \ldots, n, h_{i j}$ should satisfy the following conditions:

$$
\begin{gathered}
h_{i j}^{\sigma(\beta)}+h_{j i}^{\sigma(\beta)}=1, \quad h_{i i}=0.5, \# h i j=\# h_{j i}, \quad \text { and } \\
h_{i j}^{\sigma(\beta)}<h_{i j}^{\sigma(\beta+1)}, \quad h_{j i}^{\sigma(\beta+1)}<h_{j i}^{\sigma(\beta)}, i<j,
\end{gathered}
$$

being $h_{i j}^{\sigma(\beta)}$ the $\beta$ th smallest value in $h_{i j}$ and $h_{i j}^{\tau(\beta)}$ the $\beta$ th greater value in $h_{j i}$.

\subsection{Consensus Measure in Hesitant Fuzzy Sets}

The idea here is to extend the notion of consensus measures from the unitary interval $[0,1]$ to THFE. Analyzing the works in the literature, studies about consensus measure applied to THFS are minimal, as it is briefly addressed in the following related works.

In [13], Farhadinia investigates the relationship between entropy, similarity measure and distance measure for HFS and intervalvalued hesitant fuzzy sets (IVHFS). Beliakov et al, in [6], focus on the problem of constructing functions that are able to measure the degree of consensus for a set of inputs provided over the unit interval, from aggregation functions and fuzzy implications.

In [42], Zhang et al. present a consensus support model for GDM with hesitant fuzzy information. The hesitant fuzzy decision matrix is aggregated by using the additive aggregation (AA) operator and, in the selection process, based on the consentaneous group decision matrix, the additive weighted aggregation (AWA) operator is used to derive the overall attribute values of alternatives, by which the most desirable alternative can be found out.

Rodriguez and Martinez, in [26], provide a new consensus model for GDM problems dealing with Hesitant Fuzzy Linguistic Term Sets (HFLTS) that have been proposed to deal with hesitancy in linguistic GDM problems. In [15], Gonzales-Arteaga et al. study a different approach to the measurement of consensus based on Pearson correlation coefficient. The new correlation consensus degree measures the concordance between the intensities of preference for pairs of alternatives as expressed by the experts.

Ding et al., in [10], developed two methods for hesitant fuzzy multiple criteria GDM with group consensus in which all the experts use hesitant fuzzy decision matrices (HFDM) to express their preferences. The purpose is to present two new consensus models applied in different GDM situations.

Observe that in all these related works, none have introduced an axiomatic expression for the theoretical definition of consensus measure in THFS.

\section{Typical Hesitant Fuzzy Sets}

When the membership degree of each of the elements is given by a finite and non-empty subset of [0, 1], HFS are called Typical Hesitant Fuzzy sets (THFS). Relevant works in HFS actually make use of THFS or only works if the HFE are finite and non-empty.

Definition 14 [4, Def. 8] Let $\mathbb{H}$ be the set of all finite non-empty 
subsets of [0,1], and let $\chi$ be a nonempty set. A THFS A over $\chi$ is given by Eq. (4) where $h_{E}: \chi \rightarrow \mathbb{H}$.

Each $h_{E}(x) \in \mathbb{H}$ is called a typical hesitant fuzzy element of $\mathbb{H}$ (THFE) and the cardinality of $X$, i.e. the number of elements of $X$ is referred to as $\# X$. The $i^{t h}$ smallest element of a THFE $X$ will be denoted by $X^{(i)}$.

There are several proposals of orders for THFE, as for example the ones given in $[4,33,37,40,41]$. The unique consensus among all these orders is that all of them refines ${ }^{1}$ the following restrictive order on $\mathbb{H}$ :

$$
X \leq_{R H} Y \Leftrightarrow\left\{\begin{array}{l}
X=\mathbf{0}_{\mathbb{H}} ; \text { or } \\
Y=\mathbf{1}_{\mathbb{H}} ; \text { or } \\
\# X=\# Y \text { and } X^{(i)} \leq Y^{(i)}, \forall i=1, \ldots, \# X .
\end{array}\right.
$$

It is very restrictive since two THFE are comparable whenever both have the same cardinality.

Definition 15 [8] Let $\leq_{\mathbb{H}}$ be a partial order. The order $\preceq$ is called an admissible order on $\mathbb{H}$ with respect to $\leq_{\mathbb{H}}$ if: $(i) \preceq i$ is a linear order on $\mathbb{H}$ and (ii) for all $X_{1}, X_{2} \in \mathbb{H}, X_{1} \preceq X_{2}$ whenever $X_{1} \leq_{\mathbb{H}} X_{2}$ when the $\leq_{\mathbb{H}}$ is $\leq_{R H}$ we just call $\preceq$ of admissible order

One can observe that the admissible orders in the previous definition is not equivalent to the notion of admissible order for HFEs presented in [33]. In fact, it proposes a total order that is for HFEs restricted to a size $n$.

Next the most relevant articles on THFS are addressed. Note that there are no works using THFS with admissible orders. Since it could be possible to use total order to ordering the membership degree sets obtained from many specialists in decision making, it makes possible to compare more results, justifying the relevance of our proposal.

THFS were first presented by Bedregal et al. in [5]. Since then, they have been studied, exploring the properties of hesitant fuzzy connectives, providing examples and investigating extensions from other fuzzy logic approaches. Then, some works came next, such as [5], [4], [30], [14], [20], [25] [43] and [40].

Many other works in the literature only study about hesitant fuzzy sets instead of typical hesitant fuzzy sets. That is why, in this work the study focuses on THFS.

\section{Final Considerations}

THFS possessing a finite-set-valued fuzzy membership degrees called THFEs is a special kind of hesitant fuzzy sets. THFE are quite useful for MCDM in hesitant fuzzy setting. The ongoing work will explore THFS to express this membership degree through a set of THFE, which will consider the opinion given by the group of experts.

There are many studies about HFS, but not many when the subject is THFS and even less using consensus measure for THFS and also applying admissible orders for better comparison of the results obtained. The main research arise from the agreement of THFE with each other and the possibility and also reliability of

\footnotetext{
${ }^{1}$ A partial order $\leq{ }_{1}$ on a set $S$ refines another partial order $\leq_{2}$ on $S$ if $\left(S, \leq_{2}\right) \subseteq\left(S, \leq_{1}\right)$, i.e. for each $x, y \in S$ such that $x \leq_{2} y$ we have that $x \leq_{1} y$.
}

the combination of these elements into a single output, contributing with new theoretical support for GDM problems.

This is the first step in order to extend the results presented in [6], proposing THFS and applying an axiomatic expression for theoretical definition of consensus measure in THFS based on admissible orders

\section{Acknowledgement}

This work was supported by CAPES/Brasil, Brazilian Funding Agency CAPES, MCTI/CNPQ Universal (448766/ 2014-0) and PQ(310106/ 2016-8) and PqG/FAPERGS 02/2017(17/25510001207-0).

\section{References}

[1] J. Alcalde-Unzu, M. Vorsatz, Measuring consensus: Concepts, comparisons, and properties, in: E. Herrera-Viedma, J. L. García-Lapresta, J. Kacprzyk, M. Fedrizzi, H. Nurmi, S. Zadrozny (Eds.), Consensual Processes, Vol. 267 of Studies in Fuzziness and Soft Computing, Springer, 2011, pp. 195-211.

[2] S. Alonso, I. J. Pérez, F. J. Cabrerizo, E. Herrera-Viedma, A linguistic consensus model for web 2.0 communities, Appl. Soft Computing 13 (1) (2013) 149-157.

[3] K. Atanassov, Intuitionistic fuzzy sets, Fuzzy Sets and Systems 20 (1986) 87-96.

[4] B. R. C. Bedregal, R. Reiser, H. B. Sola, C. Lopez-Molina, V. Torra, Aggregation functions for typical hesitant fuzzy elements and the action of automorphisms, Inf. Sciences 255 (2014) 82-99.

[5] B. R. C. Bedregal, R. H. N. Santiago, H. Bustince, D. Paternain, R. Reiser, Typical hesitant fuzzy negations, Int. J. of Intell. Systems 29 (6) (2014) 525-543.

[6] G. Beliakov, T. Calvo, S. James, Consensus measures constructed from aggregation functions and fuzzy implications, Know.-Based Systems 55 (2014) 1-8.

[7] H. Bustince, E. Barrenechea, M. Pagola, J. Fernandez, Z. Xu, B. Bedregal, J. Montero, H. Hagras, F. Herrera, B. De Baets, A historical account of types of fuzzy sets and their relationships, IEEE Trans. Fuzzy Systems 24 (1) (2016) 179-194.

[8] H. Bustince, J. Fernandez, A. Kolesárová, R. Mesiar, Generation of linear orders for intervals by means of aggregation functions, Fuzzy Sets and Systems 220 (2013) 69-77.

[9] D. M. Dalalah, M. Hayajneh, F. Batieha, A fuzzy multicriteria decision making model for supplier selection, Expert Syst. Appl 38 (7) (2011) 8384-8391.

[10] J. Ding, Z. Xu, H. Liao, Consensus-reaching methods for hesitant fuzzy multiple criteria group decision making with hesitant fuzzy decision making matrices, Frontiers of IT \& EE 18 (11) (2017) 1679-1692.

[11] D. Dubois, H. Prade, Fuzzy Sets and Systems: Theory and Applications, Academic Press, NY, 1980.

[12] B. Erdamar, J. L. García-Lapresta, D. Pérez-Román, M. R. Sanver, Measuring consensus in a preference-approval context, Inf. Fusion 17 (2014) 14-21. 
[13] B. Farhadinia, Information measures for hesitant fuzzy sets and interval-valued hesitant fuzzy sets, Inf. Sciences 240 (2013) 129-144.

[14] B. Farhadinia, Study on division and subtraction operations for hesitant fuzzy sets, interval-valued hesitant fuzzy sets and typical dual hesitant fuzzy sets, J. of Intell. and Fuzzy Systems 28 (3) (2015) 1393-1402.

[15] T. González-Arteaga, R. de Andrés Calle, F. Chiclana, A new measure of consensus with reciprocal preference relations: The correlation consensus degree, Know.-Based Systems 107 (2016) 104-116.

[16] E. Herrera-Viedma, S. Alonso, F. Chiclana, F. Herrera, A consensus model for group decision making with incomplete fuzzy preference relations, IEEE Trans. Fuzzy Systems 15 (5) (2007) 863-877.

[17] E. Herrera-Viedma, F. J. Cabrerizo, J. Kacprzyk, W. Pedrycz, A review of soft consensus models in a fuzzy environment, Inf. Fusion 17 (2014) 4-13.

[18] C. Kahraman, B. Öztaysi, S. Ç. Onar, A comprehensive literature review of 50 years of fuzzy set theory, Int. J. Comput. Intell. Syst. 9 (sup1) (2016) 3-24.

[19] E. P. Klement, R. Mesiar, E. Pap, Triangular norms, Vol. 8, Springer Science \& Business Media, 2013.

[20] A. Mahmoudi, S. Sadi-Nezhad, A. Makui, M. R. Vakili, An extension on promethee based on the typical hesitant fuzzy sets to solve multi-attribute decision-making problem, Kybernetes 45 (8) (2016) 1213-1231.

[21] A. Mardani, M. Nilashi, E. K. Zavadskas, S. Awang, H. Zare, N. M. Jamal, Decision making methods based on fuzzy aggregation operators: Three decades review from 1986 to 2017, Int. J. of Information Technology and Decision Making 17 (2) (2018) 391-466.

[22] F. Mata, L. Martínez-López, E. Herrera-Viedma, An adaptive consensus support model for group decision-making problems in a multigranular fuzzy linguistic context, IEEE Trans. Fuzzy Systems 17 (2) (2009) 279-290.

[23] I. J. Pérez, F. J. Cabrerizo, E. Herrera-Viedma, Group decision making problems in a linguistic and dynamic context, Expert Syst. Appl 38 (3) (2011) 1675-1688.

[24] G. Qian, H. Wang, X. Feng, Generalized hesitant fuzzy sets and their application in decision support system, Know.Based Systems 37(1) (2013) 357-365.

[25] R. Rodríguez, B. Bedregal, H. Bustince, Y. Dong, B. Farhadinia, C. Kahraman, L. Martínez, V. Torra, Y. Xu, Z. Xu, F. Herrera, A position and perspective analysis of hesitant fuzzy sets on information fusion in decision making. Towards High Quality Progress, Inf. Fusion 29 (C) (2016) 8997.

[26] R. M. Rodríguez, L. Martínez, A consensus model for group decision making with hesitant fuzzy linguistic information, in: ISKE, IEEE, 2015, pp. 540-545.

[27] R. M. Rodríguez, Y. Xu, L. Martínez-López, F. Herrera, Exploring consistency for hesitant preference relations in decision making: Discussing concepts, meaning and taxonomy, Multiple-Valued Logic and Soft Computing 30 (2-3) (2018) 129-154.
[28] A. Rothstein, C. Butler (Eds.), On Conflict and Consensus: A Handbook on Formal Consensus Decision Making, Takoma Park, 2006.

[29] H. Santos, B. Bedregal, R. Santiago, H. Bustince, Typical hesitant fuzzy negations based on Xu-Xia-partial order, in: 2014 IEEE Conf. Norbert Wiener in the 21st Century (21CW), 2014, pp. 1-6.

[30] H. S. Santos, B. R. C. Bedregal, R. H. N. Santiago, H. Bustince, E. B. Tartas, Construction of typical hesitant triangular norms regarding Xu-Xia-partial order, in: 2015 Conference of the Int. Fuzzy Systems Association and the European Society for Fuzzy Logic and Technology (IFSAEUSFLAT-15), Gijón, Spain, 2015.

[31] V. Torra, Hesitant fuzzy sets, Int. Journal of Intell. Systems 25 (2010) 529-539.

[32] P. Victor, C. Cornelis, M. D. Cock, E. Herrera-Viedma, Practical aggregation operators for gradual trust and distrust, Fuzzy Sets and Systems 184 (1) (2011) 126-147.

[33] H. Wang, Z. Xu, Admissible orders of typical hesitant fuzzy elements and their application in ordered information fusion in multi-criteria decision making, Inf. Fusion 29 (2016) 98104.

[34] M. Xia, Z. Xu, Hesitant fuzzy information aggregation in decision making, Int. Journal of App. Reasoning 52 (2010) 395-407.

[35] M. Xia, Z. Xu, On consensus in group decision making based on fuzzy preference relations, in: E. Herrera-Viedma, J. L. García-Lapresta, J. Kacprzyk, M. Fedrizzi, H. Nurmi, S. Zadrozny (Eds.), Consensual Processes, Vol. 267 of Studies in Fuzziness and Soft Computing, Springer, 2011, pp. 263-287.

[36] M. Xia, Z. Xu, Managing hesitant information in GDM problems under fuzzy and multiplicative preference relations, Int. J. of Uncertainty, Fuzziness and Know.-Based Systems 21 (6) (2013) 865-898.

[37] Z. Xu, M. Xia, Distance and similarity measures for hesitant fuzzy sets, Inf. Sciences 181 (11) (2011) 2128-2138.

[38] L. Zadeh, Fuzzy sets, Inf. and Control 8 (1965) 338-353.

[39] L. A. Zadeh, The concept of a linguistic variable and its application to approximate reasoning - I, Inf. Sciences 8 (1975) 199-249.

[40] H. Zhang, S. Yang, Inclusion measure for typical hesitant fuzzy sets, the relative similarity measure and fuzzy entropy, Soft Computing 20 (4) (2016) 1277-1287.

[41] H. Y. Zhang, S. Y. Yang, Typical hesitant fuzzy rough sets, in: 2015 Int. Conference on Machine Learning and Cybernetics (ICMLC), Vol. 1, 2015, pp. 328-333.

[42] Z. Zhang, C. Wang, X. Tian, A consensus model for group decision making with hesitant fuzzy information, Int. J. of Uncertainty, Fuzziness and Know.-Based Systems 23 (3) (2015) 459-480

[43] N. Zhao, Z. Xu, Z. Ren, On typical hesitant fuzzy prioritized "or" operator in multi-attribute decision making, Int. J. of Intell. Systems 31 (1) (2016) 73-100.

[44] B. Zhu, Z. Xu, A fuzzy linear programming method for group decision making with additive reciprocal fuzzy preference relations, Fuzzy Sets and Systems 246 (2014) 19-33. 